\title{
Determination of centrality in nucleus-nucleus collisions at the MPD/NICA installation
}

\author{
A. Kurepin ${ }^{1, *}, A$. Litvinenko $^{2}$, and $E$. Litvinenko ${ }^{2}$ \\ ${ }^{1}$ Institute for Nuclear Research RAS, Moscow, Russia \\ ${ }^{2}$ Joint Institute for Nuclear Research, Dubna, Russia
}

\begin{abstract}
Measurement of the number of spectators in nucleus-nucleus collisions could be used to determine the number of nucleons participating in the interaction, i.e. get information about collision centrality. However, at energies of the NICA collider energy resolution of the forward hadron calorimeter is insufficient for separation of the amplitudes corresponding to different number of spectators. Uncertainty in the number of spectators leads to a large error in determining the centrality. For central events inaccuracy in determining the collision parameter is about $40 \%$, for peripheral ones, no better than $30 \%$ at a beam energy of $2.5 \mathrm{~A} \times \mathrm{GeV}$. The multiplicity scintillator detector will allow to obtain more accurate data. A certain method is proposed for determining the centrality, taking into account the real MPD installation geometry.
\end{abstract}

\section{Introduction}

The concept of centrality in the collision of relativistic heavy nuclei is an important idea that plays the most fundamental role in the collision of nuclear matter with a high baryon density, i.e. at the energies of the experimental FAIR and NICA complexes. In this case, the role of multiple rescattering and secondary processes with the production of pions and kaons is much smaller, and it can be expected that the impact parameter more clearly defines the centrality of the collision, i.e. the role of fluctuations due to the secondary interactions is much smaller. The observed particle yields due to collisions of nuclei can be more definitely associated with the magnitude of the impact parameter, i.e. with the centrality of the collision.

It is obvious that the expected formation of a new type of nuclear matter, quark gluon plasma, is more likely in more central collisions. Therefore, a more precise definition of the centrality will allow one to separate the formation of the quark gluon plasma and the secondary peripheral interactions of nuclei. On the other hand, a higher baryon density in the region of overlapping collisions of nuclei imposes a restriction on the multiplicity of particles produced which can significantly reduce the statistical accuracy of the determination of the centrality and the impact parameter.

The most common method for determining the centrality, which is used both when working on a fixed target and at colliders, is to measure the multiplicity of charged particles produced in a collision. The resulting distribution can be divided to several intervals by the magnitude of the multiplicity in each event from central to peripheral. The form of the obtained dependence, as shown in Fig. 1, for the collision of xenon nuclei at the ALICE facility

\footnotetext{
*e-mail: kurepin@inr.ru
} 
and the $\mathrm{AD}$ detector, is well described by using the Glauber model. Similar multiplicity distributions were obtained for each run of lead collisions using the V0 scintillator detector.

As another method for determining the centrality, it was proposed to register noninteracting nucleon-spectators using a hadron calorimeter. In this case, the number of interacting nucleons is obtained as the difference between the number of nucleons in the incident nucleus and the number of spectators. This method is the most widely used when working with fixed targets, for example, in the experiment NA61.

To simulate such type of forward hadron calorimeters, it is necessary to use programs which take into account the formation of spectators in the collision of nuclei and their passage through nuclear matter. Based on these programs, one can point to LAQGSM [1] used in this work and SHIELD [2].

\section{run 280234 CINT7ZAC-B \& $A_{\text {AND }}$ events}

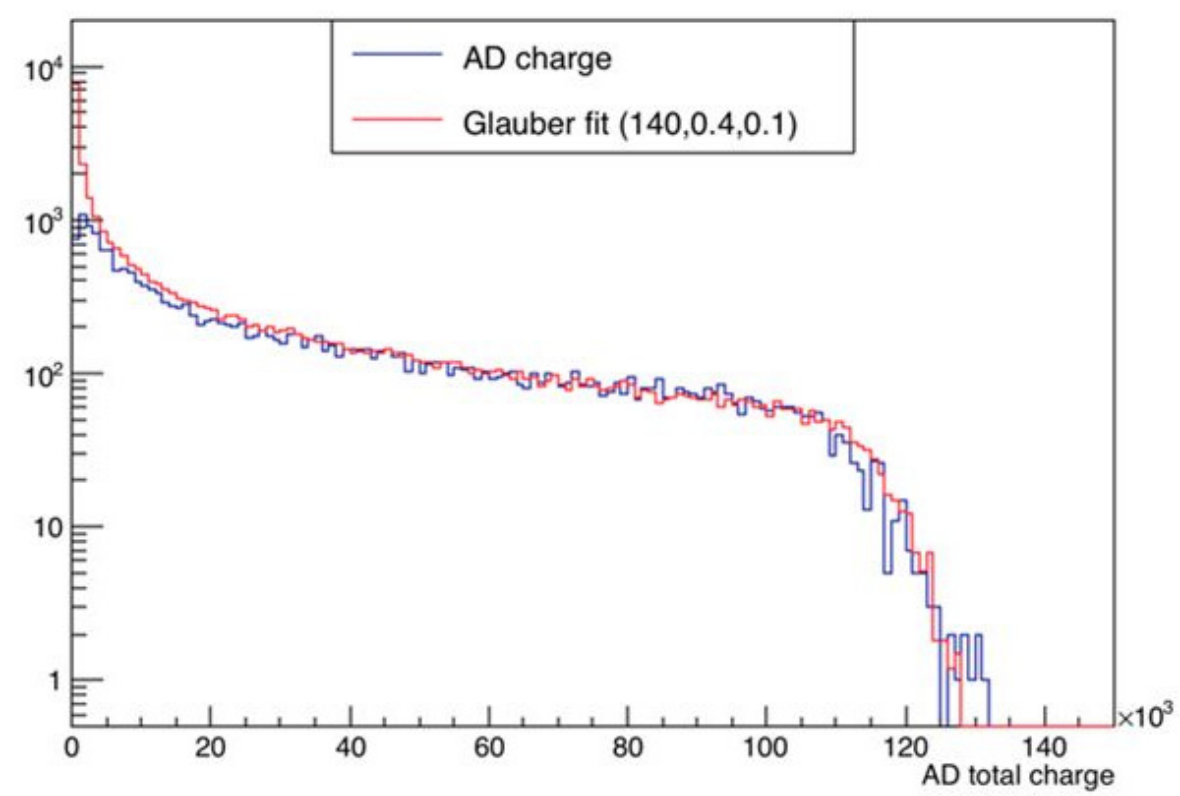

Figure 1. Multiplicity distribution for $\mathrm{Xe}-\mathrm{Xe}$ collisions at $5 \mathrm{TeV}$

\section{Centrality determination at the MPD/NICA}

The use of the forward hadron calorimeter at intermediate energy colliders, as it was first shown in [3], presents difficulties associated with the ambiguity of the information obtained. The presence of a hole in the calorimeter for the passage of the beam leads to the fact that part of the spectators goes into the vacuum tube of the collider. Fig. 2 shows the dependence of the spectator yield on the impact parameter. One can see that ambiguity is observed in the dependence of the yield on the impact parameter, which does not allow one to determine the degree of the centrality by the magnitude of the measured amplitude of the spectators. In the article [4], a method is proposed for eliminating ambiguity by determining the asymmetry of the yield of spectators according to the data of the internal and external part of the calorimeter.

Another problem when using the forward hadron calorimeter to determine the centrality at energies up to $20 \mathrm{GeV}$ is the lack of accuracy in determining the impact parameter. Indeed, to 


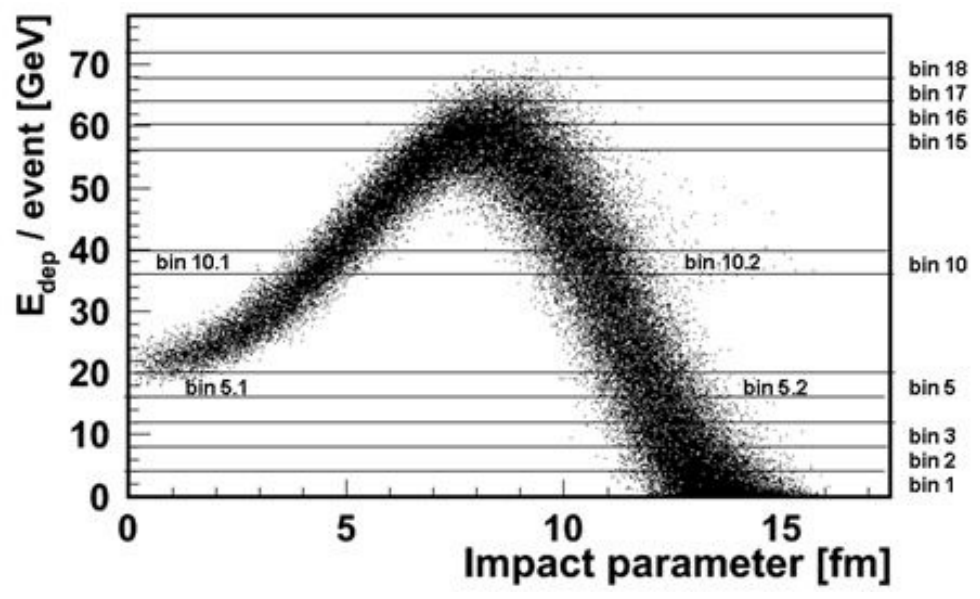

Figure 2. Dependence of total energy of spectator deposited in calorimeter on the impact parameter
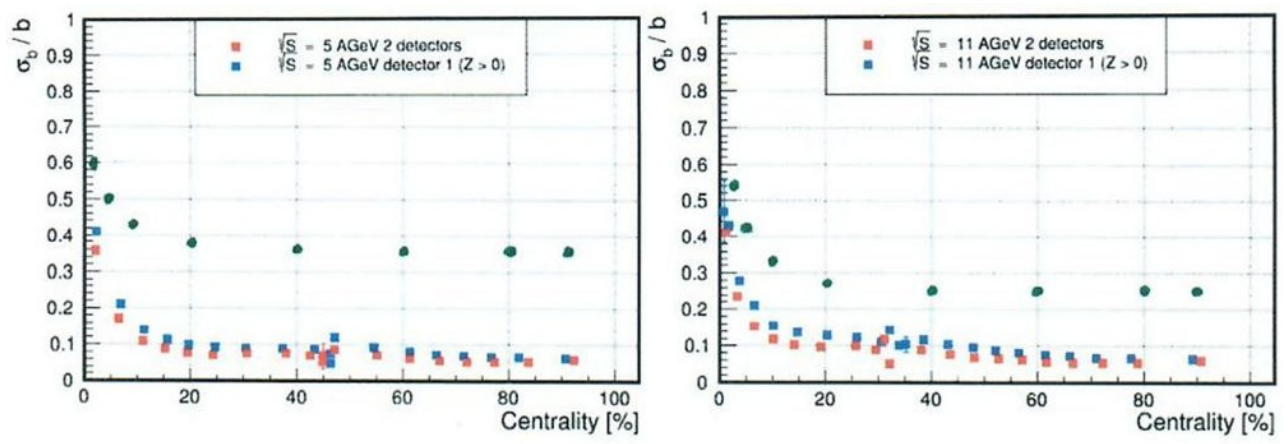

Figure 3. Resolution of impact parameter obtained in separate bins of the energy depositions in FHCal for beam energy $2.5 \mathrm{GeV}$ (left) and $5.5 \mathrm{GeV}$ (right). Blue and red points correspond to the estimate with fixed number of spectators. Green points take into account the fluctuation of spectators number

measure the number of interacting nucleons, as mentioned earlier, it is necessary to determine the number of spectators in each collision. Insufficient energy resolution of the calorimeter, obtained from calibration results for single muons and protons, does not allow one to separate of the amplitudes, corresponding to the individual particles, i.e. determine their number in each collision. This number can be obtained from the calorimeter total absorbed energy value.

In that case, when the resolution allows to separate individual spectators, i.e. determine their number in each event, the relative error of the number of spectators is proportional to $1 / \sqrt{N}$, and the same dependence of the error is in determining the centrality or impact parameter $b$.

However, the impossibility of improving the resolution of the calorimeter does not allow one to observe maxima in the amplitude of the calorimeter signal corresponding to a different number of spectators in the event, even at NA61 $(20-70 \mathrm{GeV})$. In this case, under conditions of insufficient resolution, particularly at the lower NICA energies $(2.5-5.5 \mathrm{GeV})$, the number of spectators can be found from the value of the amplitude of the calorimeter signal, which corresponds to the total energy $E$ absorbed in the calorimeter from some average number of 

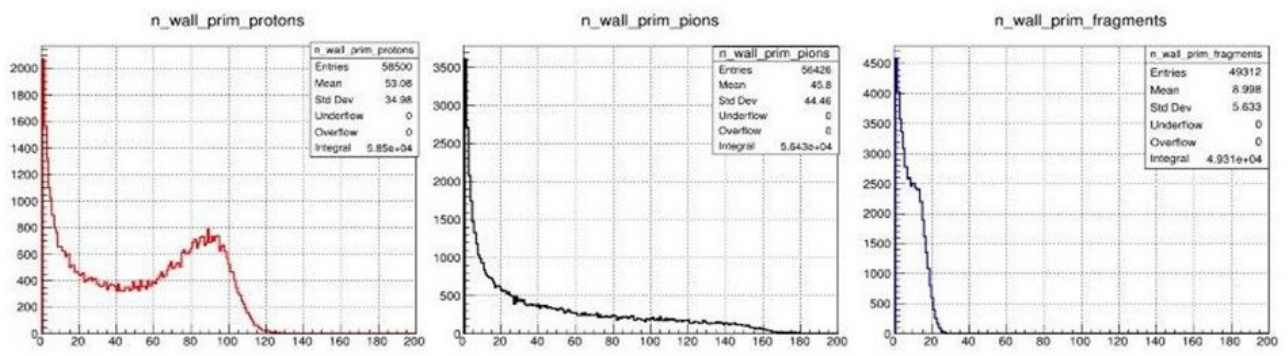

Wed Sep 12 15:14:23 2018
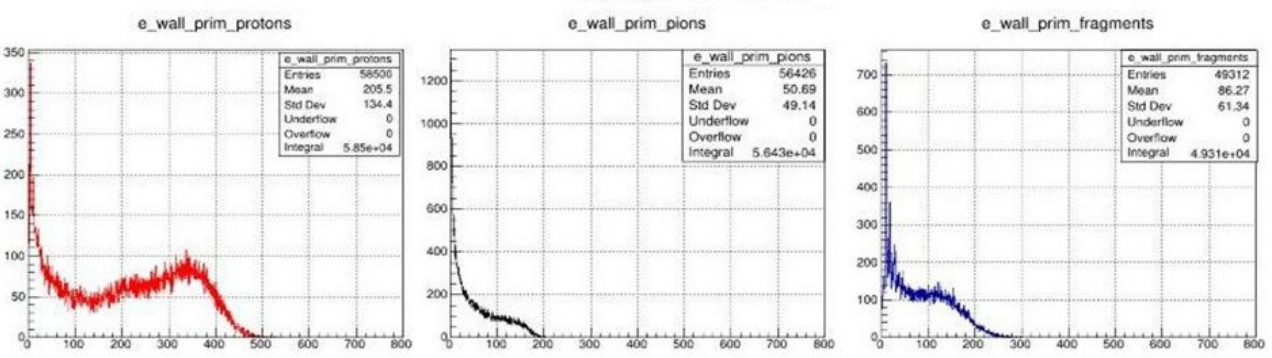

Figure 4. Multiplicity distributions for protons, pions and fragments for $5.5 \mathrm{~A} \mathrm{GeV}$ beam of $\mathrm{Au}$ ( upper part ) and total energy distribution in $\mathrm{GeV}$ (lower part)

$N$ spectators at a certain value of the impact parameter $b$ and at the average value $E(s)$ of the energy released in the calorimeter per spectator

$$
E=\sum E_{i} \approx N \times E_{s} .
$$

According to the calibration results for single muons and protons, the resolution is:

$$
\Delta E_{s} / E_{s}=0.56 / \sqrt{E_{s}} .
$$

For the number of spectators in the event it is accepted that

$$
N_{s}=E / E_{s 0},
$$

where $E_{s 0}$ is the energy of a single beam nucleon absorbed in the calorimeter.

To determine the accuracy of $N_{s}$ and the corresponding parameter $b$, one have to find an error in determining the total energy $E$. The error of product is:

$$
\Delta E=\partial E / \partial N \times \Delta N+\partial E / \partial E_{s} \times \Delta E_{s}=E_{s} \times \Delta N+N \times \Delta E_{s} .
$$

Neglecting the products of various random errors, since

$$
\lim \left(\Delta N \times \Delta E_{s}\right) \rightarrow 0,
$$

we obtain the square of the mean-square error of the total energy:

$$
(\Delta E)^{2}=E_{s}^{2} \times \Delta N^{2}+N^{2} \times \Delta E_{s}^{2}
$$

The relative error in determining the total energy can be found from the previous expression by dividing to the factor $E^{2} \approx\left(N \times E_{s}\right)^{2}$ :

$$
(\Delta E / E)^{2}=(\Delta N / N)^{2}+\left(\Delta E_{s} / E_{s}\right)^{2} .
$$



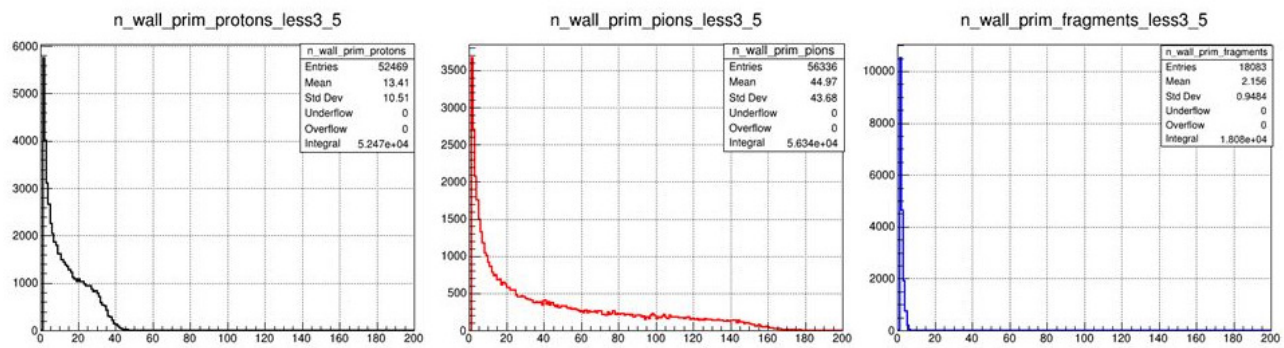

Fri Sep 14 14:30:12 2018
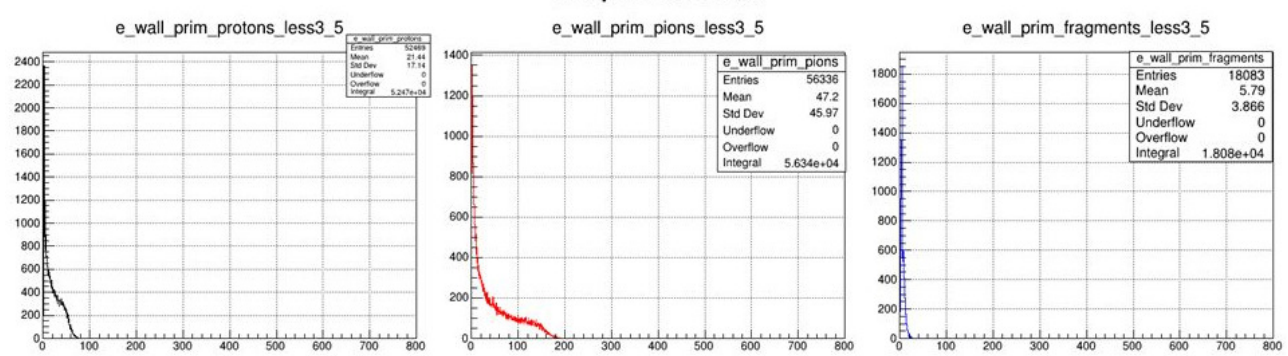

Figure 5. Same multiplicity and energy distributions for protons, pions and fragments as in Fig. 4, with condition that all charged particle with energy larger than $3.5 \mathrm{GeV}$ are removed by anticoincidence with hadron calorimeter

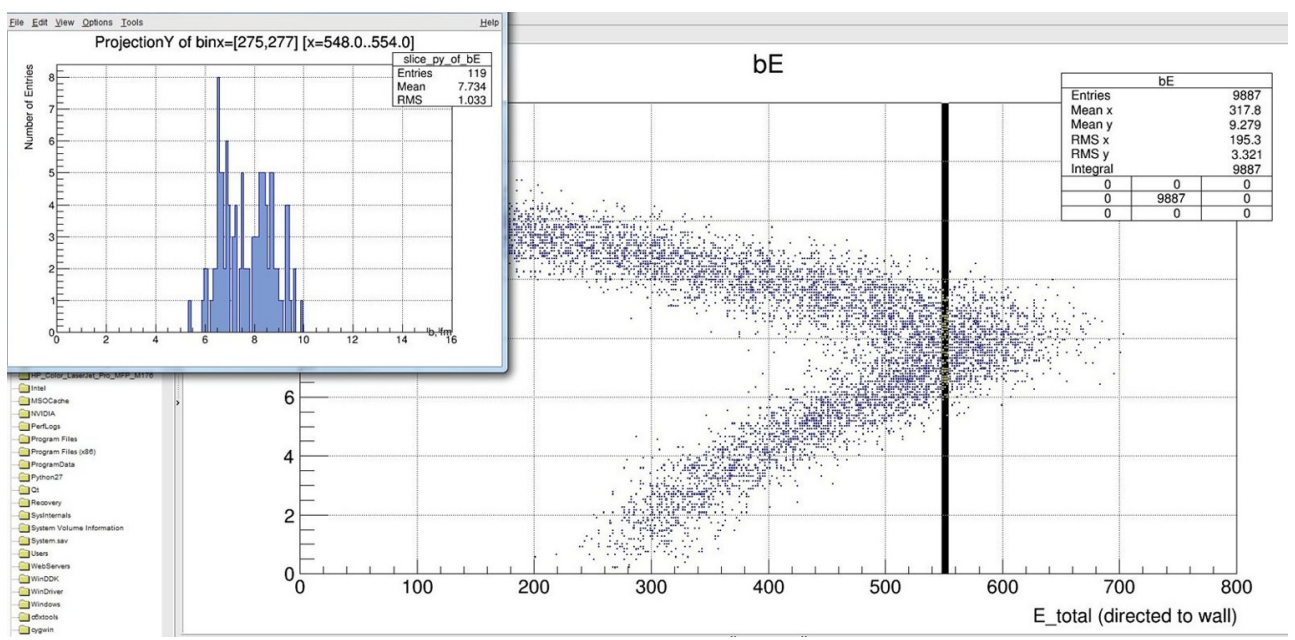

Figure 6. 6 Impact parameter distribution for fixed value $550 \mathrm{MeV}$ absorbed in the calorimeter

According to Poisson distribution

$$
\Delta N / N=1 / \sqrt{N}
$$

Taking into account the resolution for a single spectator (2), we obtain the relative error in determination of the number of spectators $N_{s}=E / E_{s 0}$

$$
\Delta N_{s} / N_{s}=\Delta E / E=\sqrt{1 / N^{2}+\left(0.56 / \sqrt{E_{s}}\right)^{2}} .
$$




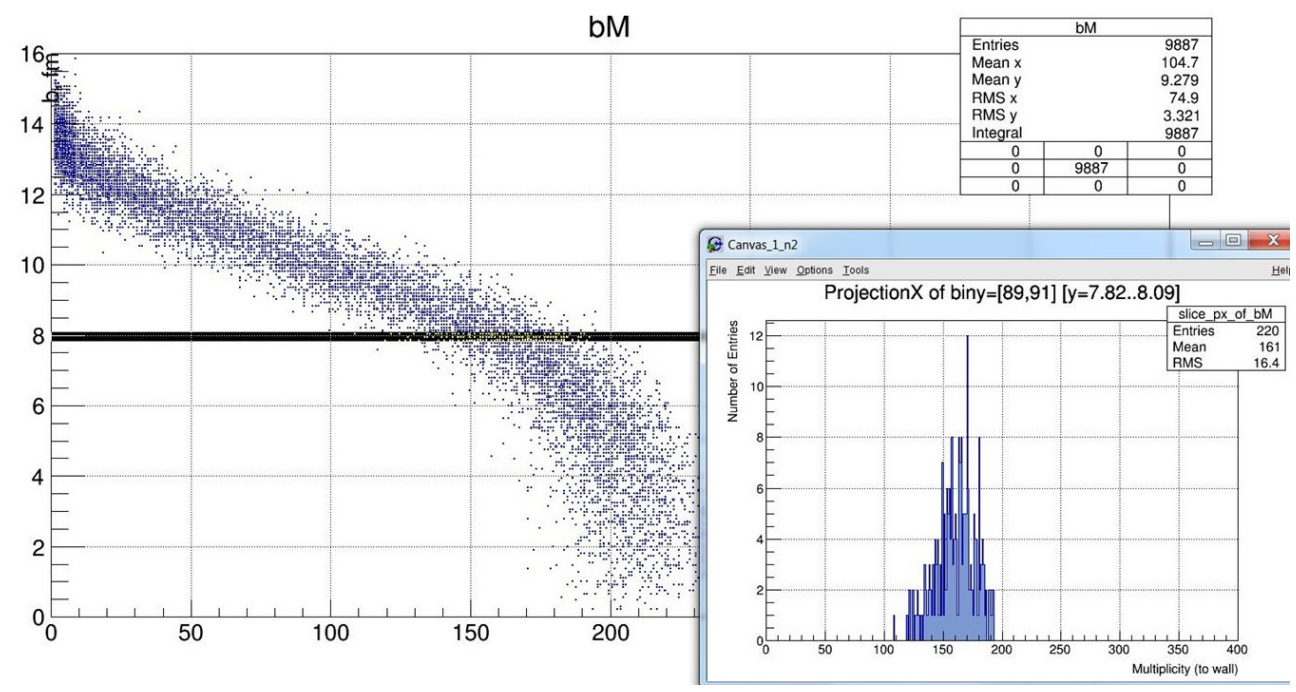

Figure 7. Multiplicity distribution for fixed value $8 \mathrm{fm}$ of the impact parameter

Thus, FHCal at the MPD/NICA gives the determination of the centrality with an accuracy of about $47 \%$ for central and $36 \%$ for peripheral events at the energy $\sqrt{s}=5 \mathrm{GeV}$. At the same time, an additional error for peripheral events is introduced due to the loss of a part of the spectators at small emission angles.

Thus, the determination of the centrality with the required accuracy in the whole interval of the MPD/NICA energies with the help of the calorimeter FHCal is impossible. For the upgrade the FMD (Forward Multiplicity Detector), a sector scintillator detector of multiplicity, should be installed, which allows one to determine the impact parameter $b$ with an accuracy better than $10 \%$ in the whole range of the centrality.

Fig. 3 shows the result of calculating the relative error in determining the collision parameter depending on the centrality of the interaction, which is divided into 5 intervals corresponding to the magnitude of the signal amplitude in the hadron calorimeter.

As shown in [5], the method of determining the centrality from the multiplicity of events cannot also be directly used at the NICA energies. Due to the relatively large transverse momentum of the spectators compared to the longitudinal one, some spectators enter the multiplicity detector what leads to the superposition of the number of the most central and most peripheral events. Fig. 4 shows the results of calculating the distribution of the multiplicity and total energy by the program LAQGSM separately for protons, which are mainly spectators, pions and fragments with a charge of more than two for the scintillator detector with outer radius $70 \mathrm{~cm}$ and inner $6 \mathrm{~cm}$. It is seen that only for pions there is no distortion of the spectrum, and this distribution can be used to determine the centrality, provided that the spectrum of charged particles is cleared from the spectators.

A certain method is proposed for eliminating spectators by incorporating an anticoincidence of the multiplicity detector with a hadron calorimeter. Pions mostly have energies less than $1.5 \mathrm{GeV}$. Therefore, setting the anti-coincidence threshold at $3.5 \mathrm{GeV}$ (see Fig. 5) almost completely removes spectators, practically without changing the pion spectrum. At the same time, there is no need to achieve high resolution of the calorimeter, and the length of its modules can be reduced. Accordingly, the weight of the calorimeter on each side of 9 tons, which forms additional difficulties for the assembly and disassembly of the installation 
can be significantly reduced. The accuracy of determining the collision parameter at various centralities is determined by the multiplicity mainly of pions, and is no worse than 10 percent even at an energy of $5 \mathrm{GeV}$.

As mentioned earlier, due to secondary collisions during the passage of produced particles through nuclear matter, a large inaccuracy arises in determining the impact parameter. Fig. 6 shows the spread of values of the impact parameter at a fixed value of the energy of the spectators, and Fig. 7 at the multiplicity of the event with the fixed value of the impact parameter. It can be seen that the error in both cases is about 10 percent.

\section{Conclusion}

In conclusion, it can be argued that in order to reliably and accurately determine the centrality and the impact parameter, the MPD installation should also have a scintillator multiplicity detector installed in front of the hadron calorimeter.

\section{References}

[1] S.G. Mashnik et al., Phys. Rev. C 95, 034613 (2017)

[2] A. Krylov et al., Phys.Part.Nucl.Lett. 11, 549-551 (2014)

[3] M.B. Golubeva et al., Yad. Fiz. 76, 2 (2013)

[4] M. Golubeva et al., TDR Forward Hadron Calorimeter FHCal (2018)

[5] M.B. Golubeva, A.P. Ivashkin and A.B. Kurepin, EPJ WoC 138, 11001 (2017) 